\title{
M. CRAMER
}

\section{Réponse de M. Cramer au commentaire de M. Rey}

Revue française d'automatique, d'informatique et de recherche opérationnelle. Recherche opérationnelle, tome 9, $\mathrm{n}^{\circ}$ V3 (1975), p. 95-96.

<http://www.numdam.org/item?id=RO_1975_9_3_95_0>

(C) AFCET, 1975, tous droits réservés.

L'accès aux archives de la revue « Revue française d'automatique, d'informatique et de recherche opérationnelle. Recherche opérationnelle » implique l'accord avec les conditions générales d'utilisation (http://www.numdam.org/ legal.php). Toute utilisation commerciale ou impression systématique est constitutive d'une infraction pénale. Toute copie ou impression de ce fichier doit contenir la présente mention de copyright.

\section{Numdam}

Article numérisé dans le cadre du programme

Numérisation de documents anciens mathématiques

http://www.numdam.org/ 
R.A.I.R.O.

(9' année, octobre 1975, V-3, p. 95 à 96)

\section{RÉPONSE DE M. CRAMER ( $\left.{ }^{1}\right)$ AU COMMENTAIRE DE $M$. REY}

Consulté au sujet de la gestion informatique de stocks industriels d'entretien et constatant la faible efficacité des programmes usuels pour contrôler ce type de stocks, j'ai été conduit à rechercher et à mettre au point un programme spécifique qui comprenait en particulier une méthode d'estimation robuste d'un fractile à partir d'une série chronologique. Partant de recherches sur la surveillance continue des électrocardiogrammes, M. Rey en est venu à définir et à étudier cette même méthode : simple et belle rencontre !

Je sais gré à $M$. Rey d'avoir complété ma liste de références par ses propres travaux, dont je n'avais pas connaissance. Sur le fond, M. Rey oppose ses résultats ( $\S 2$ dans [4]) aux miens ( $\$ 2.2 .2$ dans [1]) au sujet de la distribution asymptotique de l'estimateur considéré. En fait, cette distribution est définie par une équation fonctionnelle qui n'admet pas de solution analytique exacte et générale. M. Rey et moi-même avons donc adopté des hypothèses plus ou moins restrictives pour obtenir des modèles approchés de cette distribution. Comme nos hypothèses ne sont pas identiques, nos modèles prennent une forme différente. M. Rey, au prix d'assez laborieuses approximations, parvient à appliquer une version de loi binomiale. Pour ma part, j'ai simplement supposé que tout déplacement de l'estimateur reste petit devant le support de $F$ (ce qui est en pratique une condition nécessaire à la précision) pour développer l'équation jusqu'au deuxième ordre par rapport à $c=\alpha+\beta$. Sans autre hypothèse, on aboutit ainsi à un modèle continu, de type normal :

$$
p(s)=\frac{B}{H(s)} e^{-(2 / c) \int_{s *}^{s}[F(x)-Q] /[H(x)] d x},
$$

avec :

$$
H(x)=Q(1-Q)+(F(x)-Q)(1-2 Q)
$$

(') Centre d'Études Supérieures du Management Public.

Revue Française d'Automatique, Informatique et Recherche Opérationnelle nº oct. 1975, V-3. 
Cette solution est d'autant plus exacte que $F$ peut être approchée par une forme quadratique sur tout intervalle $[s-\alpha, s+\beta]$. A cet égard, la solution avancée par $M$. Rey, qui requiert l'hypothèse plus forte de l'approximation linéaire, apparaît donc moins générale.

A partir de sa solution, M. Rey fournit en outre une formule de variance [équation (16) dans [4]] : $\alpha \beta /\left[2(\alpha+\beta) F^{\prime}\left(s^{*}\right)\right]$.

Notons que, d'après son propre développement, cette expression représente la variance asymptotique de l'estimateur $s$ lui-même et non la variance de la fonction $F(s)$ comme il l'écrit, par inadvertance, dans son commentaire.

On peut retrouver cette expression à partir de notre solution (12) si l'on consent à deux nouvelles approximations :

$$
\begin{gathered}
F(x) \sim Q+\left(x-s^{*}\right) \cdot F^{\prime}\left(s^{*}\right), \\
H(x) \sim Q(1-Q),
\end{gathered}
$$

(12) devient alors :

$$
p(s)=\frac{B}{Q(1-Q)} e^{-\left[\left(s-s^{*}\right)^{2}\right] /\left[c Q(1-Q) / F^{\prime}\left(s^{*}\right)\right]}
$$

D'où :

$$
\operatorname{var}(s)=c Q(1-Q) i\left[2 F^{\prime}\left(s^{*}\right)\right]=\alpha \beta /\left[2(\alpha+\beta) F^{\prime}\left(s^{*}\right)\right] \text {. }
$$

Enfin, dans le cas trivial où $Q=1 / 2$ et $F$ est uniforme sur $[0, N]$ :

- (12) fournit comme approximation une loi normale (14) de variance $c / 8 d$;

- le calcul exact donne une binomiale (8) : $P(s)=2^{-N} C_{N}^{s}$ et je donne directement (p. 25, [1]) : $\operatorname{var}(F(s))=1 / 4 N$, étant bien entendu qu'ici $F(s)=s / N$ et $\operatorname{var}(s)=N / 4$.

Ces deux résultats concordent encore avec la formule de $\mathbf{M}$. Rey puisque dans ce cas particulier $c / 2=\alpha=\beta=1$ et $1 / N=d=F^{\prime}\left(s^{*}\right)$; la divergence que M. Rey croyait déceler provient donc d'une confusion entre var $(F(s))$ et $\operatorname{var}(s)$.

En conclusion, il me semble que sur cette question les résultats de M. Rey et les miens, loin de s'opposer, se complètent dans le général et se corroborent dans le particulier. Quoiqu'il en soit, j'espère avoir contribué à mettre le typographe hors de cause.

\section{RÉFÉRENCES}

Se reporter au commentaire de M. Rey.

Directeur de la Publication : P. Bordas - Imprimerie GAUTHIER-VILLARS

Dépôt légal $n^{\circ} 029-\mathrm{N}^{\circ}$ CPPP $50305-4^{\circ}$ trimestre 1975. 